\title{
Family Planning Utilization among PLWHIV Attending TASO Masaka: Evidence from a Cross-Sectional Study
}

\author{
Kizito Benjamin Birungi ${ }^{1}$, Ayebare Angella Arigye ${ }^{1}$, Namubiru Sophia ${ }^{1}$, Nabukeera Swalha Kabogo ${ }^{1}$, \\ Hussein Mukasa Kafeero ${ }^{1,2^{*}}$ and Fred Kironde ${ }^{1,2}$ \\ ${ }^{1}$ Islamic University in Uganda, Faculty of Health Sciences, Habib Medical School, Kampala Campus P.O Box, \\ 7689, Kampala- Uganda \\ ${ }^{2}$ Islamic University in Uganda, Faculty of Health Sciences, Habib Medical School, Department of Medical \\ Microbiology, Kampala Campus P.O Box, 7689, Kampala- Uganda
}

* Corresponding Author email: husseinmukasakafeero@gmail.com

Article History

Received: 20 February 2021

Revised: 30 July 2021

Accepted: 31 July 2021

Published: 31 July 2021

Student(s)

- $\quad$ Kizito Benjamin Birungi

- Ayebare Angella Arigye

- Namubiru Sophia

- Nabukeera Swalha Kabogo

Academic Year: 2019-2020
Course Level: Bachelor
Course Name: MBChB (Bachelor of
\[ \begin{array}{l}\text { Medicine and Bachelor } \\ \text { of Surgery) }\end{array} \]
Course year: Final Year

$\operatorname{Mentor}(\mathrm{s})$

- Dr. Hussein Mukasa Kafeero

- $\quad$ Prof. Fred Kironde

\section{ABSTRACT}

People living with HIV (PLWHIV) need family planning (FP) services to avert the spread of HIV and eliminate mother to child transmission (MTCT). Besides, family planning services greatly reduce the incidence of vertical transmission reducing new cases of pediatric infections. The aim of this study was to establish the factors influencing the utilization of FP services among PLWHIV affiliated to The AIDS Service Organization (TASO) Masaka responsible for the unmet need for FP utilization. A cross-sectional study with quantitative data analysis was used. A structured questionnaire was used to collect data in line with the set objectives. Categorical data were presented as frequency percent and continuous data as a mean \pm standard deviation. The data were analyzed with the statistical software called Medcalc software v. 19.5.6. Analysis was done using the test of proportion at $95 \%$ confidence interval and a $\mathrm{P}<0.05$ was considered statistically significant. The study had significantly more females, married, and of low education level $(\mathrm{p}<0.05)$. Medium parity of 1-3, a spouse being comfortable with a partner using FP, knowledge about FP were significantly associated with FP use $(\mathrm{P}<0.05)$. The Majority of the participants were using FP methods $(\mathrm{P}<0.05)$ with injections being the most used method $(\mathrm{P}<0.05)$. The Majority of the clients would encourage others to use FP $(\mathrm{P}<0.05)$ and HIV had no effect on FP use $(\mathrm{P}<0.05)$. Culture and society beliefs did not affect FP utilization $(\mathrm{P}<0.05)$. Being close to the service provider, hospitality of the service provider, information about the benefits and risks associated with FP use were significantly associated with utilization of FP methods $(\mathrm{P}<0.05)$. However, the overall utilization of FP was low $(58.9 \%)$ and was largely affected by personal factors other than community or service delivery factors. Future research should focus on the putative personal factors that may prevent the utilization of FP methods.

Keywords: Family planning, PLWHIV, Cross-sectional, TASO 


\section{Introduction}

There were an estimated 37.7 million people living with HIV at the end of 2020, over two thirds of whom (25.4 million) are in the World Health Organization (WHO) African Region [1]. As a result of concerted international efforts to respond to HIV, coverage of services has been steadily increasing. In 2019, 68\% of adults and $53 \%$ of children living with HIV globally were receiving lifelong antiretroviral therapy (ART). A great majority $(85 \%)$ of pregnant and breastfeeding women living with HIV also received ART, which not only protects their health, but also ensures prevention of HIV transmission to their newborns [2] [3].

Uganda has 1,400,000 people living with HIV and an incidence per 1000 uninfected being 1.4 over a year in all age groups. Of these, $5.7 \%$ are of productive age with young women being most vulnerable. Besides, the high poverty levels, high fertility rates and low access to contraception services has maintained the prevalence high in this cohort [4]. Moreover, among the 1.9 billion women of reproductive age group (1549 years) worldwide, 1.1 billion have a need for family planning and of these, 842 million are using contraceptive methods and 270 million have unmet need for contraception [5].

Therefore, people living with HIV both locally and globally are in dire need of family planning services in efforts to avert the spread of HIV as well as eliminate mother to child transmission by advocating and promoting intended or planned pregnancies thereby also preventing the complications that arise from the mental, psychological and obstetric strain from these unplanned pregnancies. Besides, the family planning services greatly reduce the incidence of vertical transmission thus reducing the statistic of new and pediatric infections. Owing to improper utilization of the family planning services, the vicious cycle of unregulated fertility, vertical transmission and the new infections thus spread and more infections go on [5].

Family planning (FP) is defined as the ability of individuals and couples to anticipate and attain their desired number of children and the spacing and timing of their births achieved through the use of contraceptive methods and the treatment of involuntary infertility [2]. Family planning reduces the danger of unintended pregnancies among women living with HIV, leading to fewer infected babies and orphans [6]. To prevent unintended pregnancies among HIV positive women, provision of quality FP services is important. FP services together with preventing primary HIV infection in women have been reported to significantly reduce the proportion of infants infected with HIV. [7]

Moreover, elimination of Mother to Child Transmission (EMTCT) starts with preventing the mother from having an unintended pregnancy [6].

Scaling up FP services is one of the most effective interventions in the prevention of maternal, infant and child deaths globally[8]. Besides, family planning interventions that aid in lowering maternal, infant and child mortality, potentially contribute to the targets of many aspects of the newly developed Sustainable Development Goals (SDGs) [33]. Family planning especially in PLWHIV will drastically reduce the number of vertical transmissions which in turn reduces the number of HIV cases in the country as well as promote elimination of mother-to-child transmission which is a core health strategy for the country [6].

Owing to the increased availability of Anti-retroviral drugs among people living with HIV, the desire for conception has also increased further elevating the risk for unplanned or unintended pregnancies which without appropriate care could avert the concept of elimination of mother-to-child transmission [7]. However, with family planning practices being implemented, a desirable intended pregnancy can prevent this thus averting the risk of pediatric HIV and as such the statistics in regards to the new HIV infection [5]. The Uganda Ministry of Health guidelines advocate for the integration of family planning services in the care and treatment of HIV due to the cost effectiveness it implies when used alongside other essential services such as ARV acquisition and EMTCT [10]. Moreover, the Ugandan Ministry of Health targets to improve FP from the current $35 \%$ to $50 \%$ by 2020 in a program that started in 2016 to 2020 [10].

Elsewhere in Africa, there is still an unmet need for family planning as reported by the studies by Schrumpf et al., [11] in Ghana,Babazadeh et al., [12] in Kinshasa DRC, Derek et al, 2019 in Ethiopia [13], Ahmed et al., in South Sudan [11], Prata et al in Angola [12] and Kriel et al., in Kwazul-Natal South Africa [11] with key limitations being the putative side effects, lack of male partner involvement and social cultural factors 
Kizito et al., Adv. J. Grad. Res.; Vol. 10, Issue 1, pp: 51-61, July 2021

as well as religion [14] [15]. The aim of our study was to understand the factors associated with utilization of family planning services among people living with HIV so as to design strategies to overcome, promote and implement family planning in turn improving their health.

\section{Materials and Methods}

\subsection{Study subjects and methods}

The study was a cross-sectional study conducted on PLWHIV affiliated to TASO Masaka, one of TASO(U) limited-service centers located in the central part of Uganda between September and November 2020. By June 2018, TASO Masaka had, cumulatively (since inception), served and improved the lives of 37,000 clients 25,900 (70\% female) and 11,100 (30\% male), their families and communities. It operates within a $75 \mathrm{~km}$ radius and its catchment area covers the districts of the greater Masaka Region, including; Masaka, Lwengo, Bukomansimbi, Lyantonde, Kyotera, Rakai, Sembabule, Kalungu,and Kalangala served by 33 full time staff 14 part time staff, 100 Community AIDS Support Agents, 125 Community Client Led ART Distribution (CCLAD) peer leaders and 25 expert clients who support Centre clinics (TASO 2018). The study population involved sexually active PLWHIV getting services from TASO Masaka of 18 years and above. This included the married, single and divorced. The study sample size had 185 study participants, determined using the formula for simple random sampling using single proportions given by [16]. We randomly chose 10 clients from the available clients for the day at TASO Masaka. It's from these numbers that we assessed if they met the exclusion and inclusion criteria. If one did not meet the criteria, then we randomly chose another client. The Inclusion criteria required sexually active male or female PLWHIV getting services from TASO Masaka while the exclusion criteria included PLWHIV below 18 years, sexually active male PLWHIV whose spouse is currently pregnant or exclusively breastfeeding and female sexually active PLWHIV who is using contraceptives to manage other medical conditions

\subsection{Data collection}

Data was collected by the use of a self-administered/researcher administered structured questionnaire. Researcher administration of the questionnaire was opted for following the failure of the study participant to read and write. The questionnaire was translated to both English and Luganda ( $99.9 \%$ of the population understands at least one of the two languages). The research team pre-tested the data collection tool to a sample of thirty (30) respondents attending the family planning clinic at the same center. After pre-testing we excluded the unclear items or questions. The first segment of the questionnaire encompassed three (3) items on the details of the socio-demographic characteristics of the of the study participants, the second section contained eight (8) questions about the personal factors influencing the utilization of family planning by PLWHIV attending TASO Masaka with 'Yes' or 'No' response save for the choice of family planning method. The third section explored the community factors that influence the utilization of FP by PLWHIV attending TASO Masaka and had two (2) questions still with 'Yes' or 'No' response. The last section of the questionnaire that explored the service delivery factors influencing the utilization of FP services by PLWHIV attending TASO Masaka had four (4) questions with 'Yes' or 'No' responses except for the accessibility of the family planning services at this facility. We got responses from nearly all our targeted number of participants except for 15 questionnaires which could not be returned by the research assistant. Thus, a $92.5 \%$ questionnaire return was satisfactory to get reliable results. The data collection tool had forty-one (41) questions and it took an average of 30 to 40 minutes to be responded to. On average, 10 questionnaires were filled per day and data collection took about four (4) weeks to be completed.

\subsection{Quality control}

To ensure quality of the data collected, different measures were put in place, the questionnaires were crosschecked for any errors by the research supervisor and pretested before by using fellow colleagues to eliminate any vagueness in the questions. Additionally, all questions were explained to the participants before handing to them the questionnaires and at any point of filling the questionnaire, one of us was 
available for any enquiries. Moreover, after all questionnaires were filled, they were immediately crosschecked for any missing data and any questionnaires missing part of the data or containing unclear information were discarded.

\subsection{Data management and analysis}

Following data collection, questionnaires were kept in the folder and at the end of the day; the information was turned into soft copy and stored on a laptop. All the questionnaires for the research were kept in a box file for helpful reference in times of need. Quantitative methodology of analysis was used and the data were analyzed using a statistical software called Medcalc v.19.5.6. Bivariate analysis was performed between FP use (dependent variable) and each of the potential factors associated with FP use (independent variables), one at a time. These included personal, community and service delivery factors. Their $95 \%$ confidence intervals $(\mathrm{CI})$ and p-values were obtained. The findings at this stage helped us to identify important associations.

\section{Results}

\subsection{Socio-demographic characteristics of our study subjects}

The socio-demographic characteristics are as shown in figure 1 and table 1. Pertaining age and gender, the study participants were generally young with mean age $35.45 \pm 8.185$ and the majority were female significantly more than their male counterparts ( $\mathrm{P}<0.0001,95 \% \mathrm{C} 1=44.94 \%$ to $73.26 \%$ ). Regarding marital status, the majority of our study participants were married (55.1\%) significantly higher than those who were single $(\mathrm{P}=0.0004,95 \% \mathrm{CI}=15.24 \%$ to $46.56 \%)$, divorced $(\mathrm{P}=0.0002,95 \% \mathrm{CI}=17.26 \%$ to $49.04 \%)$ or widowed ( $\mathrm{P}=0.0138,95 \% \mathrm{CI}=11.15 \%$ to $61.68 \%$ ).

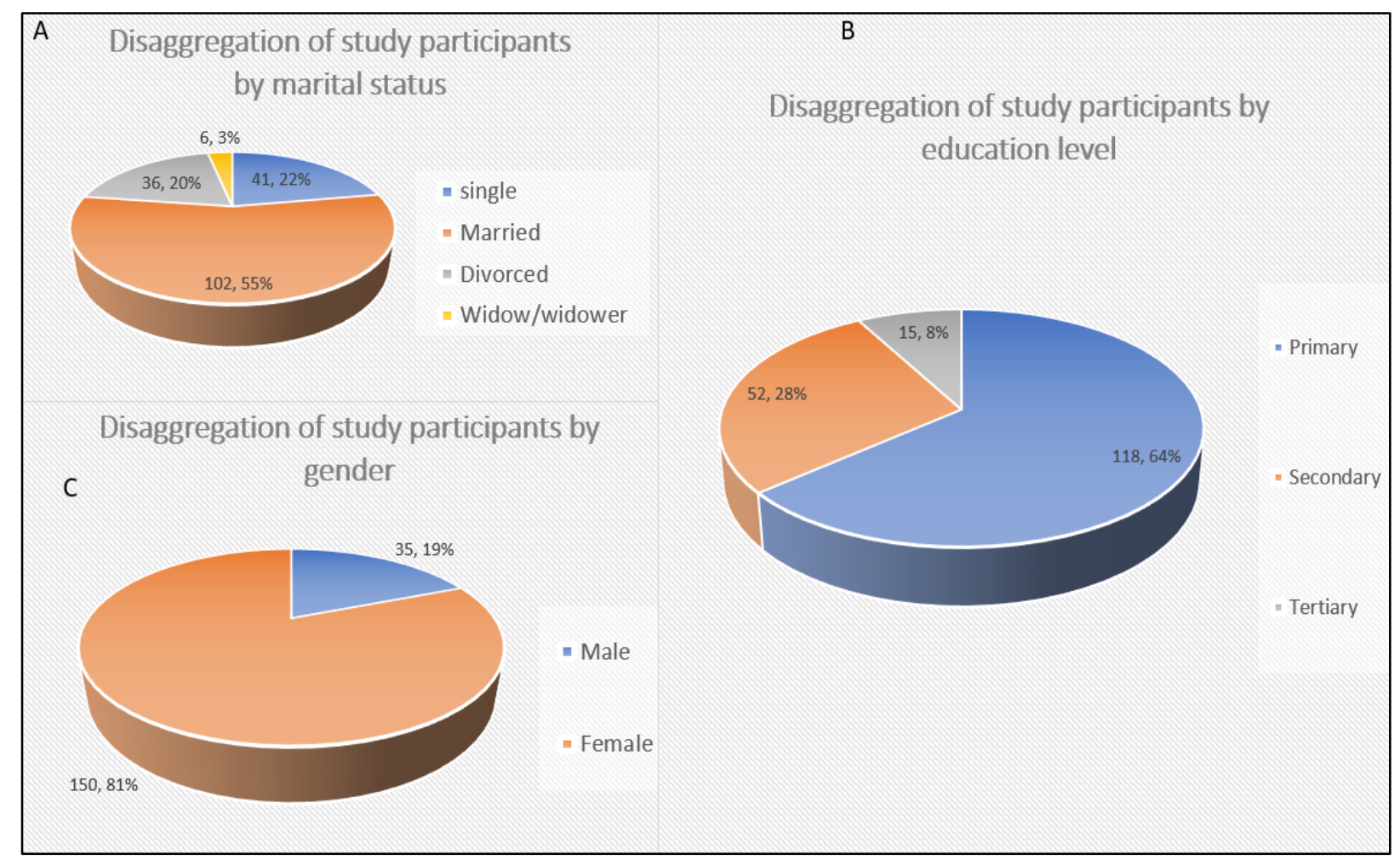

Figure1: Socio-demographic characteristics of the study participants disaggregated into the various study groups: A; Marital status, B; Education level and C; Gender

Relating to the education level, the majority of our study subjects $(63.8 \%)$ attained primary or no formal education at all significantly more than those who attained the secondary level $(\mathrm{P}<0.0001,95 \% \mathrm{CI}=19.58 \%$ to $48.87 \%$ ) or the tertiary level of education ( $\mathrm{P}<0.0001,95 \% \mathrm{CI}=30.51 \%$ to $66.06 \%$ ). (Table 1 ). 
Kizito et al., Adv. J. Grad. Res.; Vol. 10, Issue 1, pp: 51-61, July 2021

Table 1: Demographic Characteristics of the study subjects

\begin{tabular}{|c|c|c|c|}
\hline Variable & $\mathrm{N}(\%)$ & $\mathrm{P}$ value & $95 \% \mathrm{CI}$ \\
\hline Sample size, $\mathrm{n}$ & 185 & & \\
\hline Age, mean $\pm \mathrm{SD}$ & $35.45 \pm 8.185$ & & \\
\hline \multicolumn{4}{|l|}{ Gender } \\
\hline Male & $35(18.9 \%)$ & & \\
\hline Female & $150,(81.1 \%)$ & $<0.0001 *$ & $44.94 \%$ to $73.26 \%$ \\
\hline \multicolumn{4}{|l|}{ Marital status } \\
\hline single & $41(22.2 \%)$ & $0.0004 *$ & $15.24 \%$ to $46.56 \%$ \\
\hline Married & $102(55.1 \%) \operatorname{Ref}$ & & \\
\hline Divorced & $36(19.5 \%)$ & $0.0002 *$ & $17.26 \%$ to $49.04 \%$ \\
\hline Widow/widower & $6(3.2 \%)$ & $0.0138^{*}$ & $11.15 \%$ to $61.68 \%$ \\
\hline \multicolumn{4}{|c|}{ Level of education } \\
\hline Primary & $118(63.8 \%) \operatorname{Ref}$ & & \\
\hline Secondary & $52(28.1 \%)$ & $<0.0001 *$ & $19.58 \%$ to $48.87 \%$ \\
\hline Tertiary & $15(8.1 \%)$ & $<0.0001 *$ & $30.51 \%$ to $66.06 \%$ \\
\hline
\end{tabular}

Continuous data is presented as mean $\pm \mathrm{SD}, * \mathrm{P}$ significant at $<0.05, \mathrm{CI}=$ confidence interval, Categorical data is presented as frequency $(\%), \mathrm{SD}=$ Standard Deviation

\subsection{Personal factors influencing utilization of FP services by clients attending TASO Masaka}

When we analyzed the influence of the personal factors on the uptake of family planning services among the TASO Masaka clients, interesting results were obtained. First and foremost, the magnitude of parity had a strong bearing on family planning uptake. In this respect, our findings showed that having 1-3 children was significantly associated with uptake of FP at $46.0 \%$ compared to those with no child at $9.7 \%$ ( $\mathrm{P}=0.0044$, $95 \% \mathrm{CI}=12.61 \%$ to $49.07 \%$ ). Notwithstanding, the proportion of mothers who reported to have high parity ( $\geq 6$ children) and could take up family planning services were significantly fewer than those with moderate parity ( $\mathrm{P}=0.0080,95 \% \mathrm{CI}=11.6 \%$ to $51 \%$ ). Further still, we investigated the role of the influence of the partner in the uptake of family planning. Our findings revealed that the majority of the study participants (76.8\%) were comfortable with their partner partaking family planning $(\mathrm{P}<0.0001,95 \% \mathrm{CI}=37.25 \%$ to $65.43 \%$ ). Regarding knowledge of family planning use as another putative personal factor influencing the adoption of family planning, almost all our participants (99.5\%) were exclusively aware of family planning $(\mathrm{P}<0.0001,95 \% \mathrm{CI}=19.91 \%$ to $99.65 \%)$ and the majority of them $(58.9 \%)$ were indeed using family planning method $(\mathrm{P}=0.0175,95 \% \mathrm{CI}=3.16 \%$ to $31.39 \%)$. (Table 2 )

Table 2: Personal factors influencing the utilization of FP by PLWHIV attending TASO Masaka

\begin{tabular}{lccc}
\hline Study variable & Frequency $(\%)$ & P value & $95 \%$ CI \\
\hline Parity & $18(9.7 \%)$ & $0.0044 *$ & $12.61 \%$ to $49.07 \%$ \\
None & $85(46.0 \%)$ Ref & & \\
$1-3$ & $69(37.3 \%)$ & 0.2784 & $-6.89 \%$ to $23.55 \%$ \\
$4-6$ & $13(7.0 \%)$ & $0.0080^{*}$ & $11.6 \%$ to $51 \%$ \\
$>6$ & & & \\
Partner comfortability & $84(76.8 \%)$ & & $31.75 \%$ to $68.08 \%$ \\
Yes & $25(23.2 \%)$ & $<0.0001 *$ & \\
No & $184(99.5 \%)$ & & $19.91 \%$ to $99.65 \%$ \\
Heard of FP & $1(0.5 \%)$ & $<0.0001 *$ & \\
Yes & & & \\
No & & & \\
\hline
\end{tabular}




\begin{tabular}{|c|c|c|c|}
\hline \multicolumn{4}{|l|}{ Currently using } \\
\hline Yes & $109(58.9 \%)$ & & \\
\hline No & $76(41.1 \%)$ & $0.0175^{*}$ & $3.16 \%$ to $31.39 \%$ \\
\hline \multicolumn{4}{|l|}{ FP method } \\
\hline Condoms & $33(30.3 \%)$ & 0.1408 & $-5.19 \%$ to $35.34 \%$ \\
\hline Injections & $50(45.8 \%)$ Ref & & \\
\hline IUD & $5(4.6 \%)$ & 0.1273 & $-11.68 \%$ to $59.35 \%$ \\
\hline Implant & $16(14.7 \%)$ & 0.0202 & $5.63 \%$ to $50.12 \%$ \\
\hline Others & $5(4.6 \%)$ & 0.0645 & $-2.27 \%$ to $57.76 \%$ \\
\hline \multicolumn{4}{|c|}{$\begin{array}{l}\text { Encourage another } \\
\text { person }\end{array}$} \\
\hline Yes & $181(97.8 \%)$ & & \\
\hline No & $4(2.2 \%)$ & $<0.0001^{*}$ & $46.5 \%$ to 98.14 \\
\hline \multicolumn{4}{|c|}{ HIV Effect on FP Use } \\
\hline Yes & $183(98.9 \%)$ & & \\
\hline No & $2(1.1 \%)$ & $<0.0001 *$ & $32.33 \%$ to $99.15 \%)$ \\
\hline
\end{tabular}

$* \mathrm{P}$ significant at $<0.05, \mathrm{CI}=$ confidence interval, Categorical data is presented as frequency $(\%)$

As presented in figure 2, the most commonly used family planning was reported to be the injectables with a use rate of 50/109 (45.8\%) participants and the least popular was IUD with a utilization rate of 5/109 $(4.6 \%)$ participants. On analysis of the differences in the observed utilization rate, injections were significantly more used than the implant at $14.7 \%(\mathrm{P}=0.0202,95 \% \mathrm{CI}=5.63 \%$ to $50.12 \%$ ) (Table 2 ).

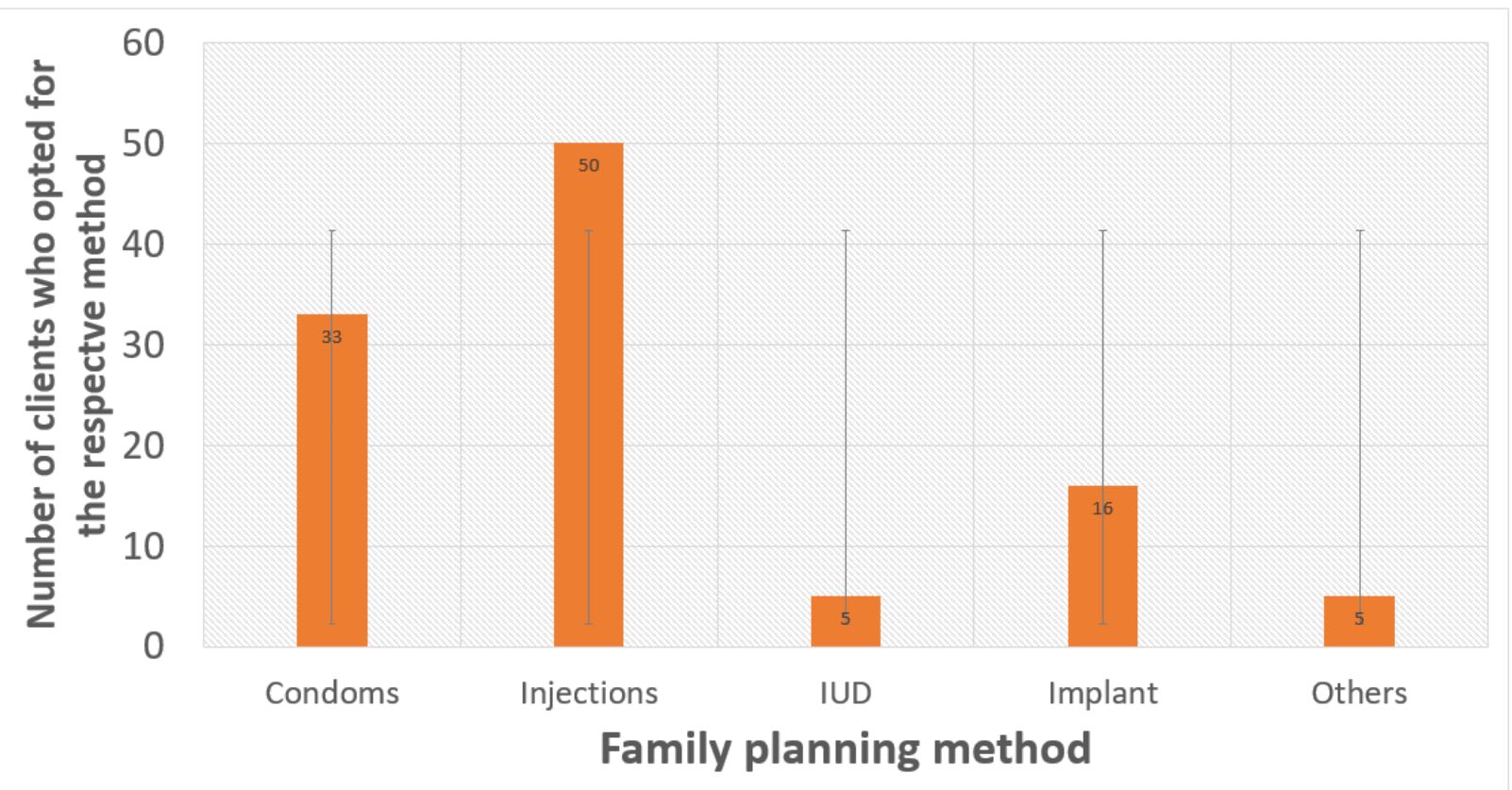

Figure 2: Different family planning methods and their relative utilization by the study participants. The error bars are standard error bars. 
Kizito et al., Adv. J. Grad. Res.; Vol. 10, Issue 1, pp: 51-61, July 2021

On investigating the influence of the partner on the uptake of a family planning method, our report showed that of the $55.7 \%$ of the participants who used family planning, $76.8 \%$ of the participants' partners were comfortable significantly more than those who were not comfortable $(\mathrm{p}<0.0001,95 \% \mathrm{CI}=31.75 \%$ to $68.08 \%$ ). Additionally, the majority of the study participants $(99.5 \%)$ would encourage their peers to use family planning $(\mathrm{P}<0.0001,95 \% \mathrm{CI}=46.5 \%$ to 98.14\%). Besides, approximately all the study subjects agreed that having HIV could not affect their desire to use a family planning method at 98.9\% $(\mathrm{P}<0.0001$, $95 \% \mathrm{CI}=32.33 \%$ to $99.15 \%$ ) (Table 2 )

\subsection{Community factors that influence the utilization of FP by PLWHIV attending TASO Masaka}

When we investigated the contribution of community factors on the uptake of the family planning method(s) by our participants in the study, fascinating results were obtained. As presented in table 3, nearly all the study subjects recruited in our study (97.3\%) were not influenced by their respective cultures in their routine uptake of the family planning services $(\mathrm{P}<0.0001,95 \% \mathrm{CI}=50.74 \%$ to $97.64 \%$ ). Similarly, 96.2\% of the study subjects were not affected by the negative society beliefs in their utilization of the family planning services $(\mathrm{P}<0.0001,95 \% \mathrm{CI}=55.88 \%$ to $96.48 \%)$.

Table 3: Community factors that influence the utilization of FP by PLWHIV attending TASO Masaka

\begin{tabular}{lccc}
\hline Study variable & Frequency (\%) & P value & 95\% CI \\
\hline Culture effect on FP & $5(2.7 \%)$ & & \\
Yes & $180(97.3 \%)$ & $<0.0001 *$ & $50.74 \%$ to $97.64 \%$ \\
No & $7(3.8 \%)$ & & \\
Society beliefs on FP & $178(96.2 \%)$ & $<0.0001 *$ & $55.88 \%$ to $96.48 \%$ \\
Yes & No &
\end{tabular}

$* \mathrm{P}$ significant at $<0.05, \mathrm{CI}=$ confidence interval, Categorical data is presented as frequency $(\%)$

\subsection{Service delivery factors influencing the utilization of FP services by PLWHIV attending TASO Masaka}

Regarding the influence of the quality-of-service delivery and their accessibility on the uptake of family planning services by our participants, the majority of them $(68.1 \%)$ were close to the service provider $(\mathrm{P}<$ $0.0001,95 \% \mathrm{CI}=20.89 \%$ to $49.08 \%$ ) so, services were easily accessible. Similarly, $99.5 \%$ of the participants reported hospitality by the family planning service providers ( $\mathrm{P}<0.0001,95 \% \mathrm{CI}=19.91 \%$ to 99.65\%). Further still, health education and literacy were effectively done to the clients receiving family planning services. To this effect, $82.2 \%$ of the participants reported to have been sensitized about the benefits of using family planning ( $\mathrm{P}<0.0001,95 \% \mathrm{CI}=46.85 \%$ to $75.21 \%$ ). Besides, $70.8 \%$ of our study participants reported to have been informed about the side effects of using the family planning services $(\mathrm{P}<0.0001$, $95 \% \mathrm{CI}=26.03 \%$ to $54.21 \%$ ) (Tables 5 ).

Table 5: Service delivery factors influencing the utilization of FP services by PLWHIV attending TASO Masaka

\begin{tabular}{lccc}
\hline Study variable & Frequency $(\%)$ & P value & $95 \%$ CI \\
\hline Accessibility & $126(68.1 \%)$ & & \\
Close & $59(31.9 \%)$ & $<0.0001 *$ & $20.89 \%$ to $49.08 \%$ \\
Far & & & \\
Hospitality by care & & & \\
givers & $184(99.5 \%)$ & $<0.0001 *$ & $19.91 \%$ to $99.65 \%$ \\
Yes & $1(0.5 \%)$ & \\
No &
\end{tabular}




\begin{tabular}{|c|c|c|c|}
\hline \multicolumn{4}{|c|}{ Informed about benefits } \\
\hline Yes & $152(82.2 \%)$ & & \\
\hline No & $33(17.8 \%)$ & $<0.0001 *$ & $46.85 \%$ to $75.21 \%$ \\
\hline \multicolumn{4}{|c|}{ Informed about risks } \\
\hline Yes & $131(70.8 \%)$ & & \\
\hline No & $54(29.2 \%)$ & $<0.0001 *$ & $26.03 \%$ to $54.21 \%$ \\
\hline
\end{tabular}

$* \mathrm{P}$ significant at $<0.05, \mathrm{CI}=$ confidence interval, Categorical data is presented as frequency $(\%)$

\section{Discussion}

The study demonstrated a high level of knowledge pertaining family planning with $99.7 \%$ of the respondents having heard of family planning. This finding is consistent with the earlier finding from the study by Habte and Namasasu [34] conducted in Malawi on Family planning use among women living with HIV during the national survey. They reported that $99.8 \%$ of their study participants were knowledgeable about FP. However, the reported use of FP among our study participants was low at $58.9 \%$ among PLWHIV at TASO Masaka consistent with the findings of Habte and colleague who reported FP non-use of $51.3 \%$ suggesting an unmet need for family planning use in Malawi and in our cohort. The finding from our study on FP utilization rate and that reported by Habte and Namasasu is in conformity with the earlier report by Nattabi B et al [17] on family planning utilization among people living with HIV in post-conflict Northern Uganda. Notwithstanding however, there has been a remarkable improvement in the utilization of FP services at TASO Masaka from an earlier 24\% uptake in a study done in 2017 [18] to 58.9\% in the current study.

The factors that influenced the use of family planning in our study were attributable to level of education, contraception method, HIV sero-positivity, Age, gender, partner's comfortability, ociety and cultural beliefs as well as service delivery factors and these have been described elsewhere [19]. The majority of our participants had low levels of education and this could explain the low utilization of family planning services since studies in Uganda and in the diaspora have implicated low levels of education in non-use of family planning [20][21].

Pertaining the method of contraception, the main method of contraception was injectable contraceptives $(45.8 \%)$ because it's easy to use, administer, and can be used without the partner's knowledge as well as easy follow up which is once every 3 months. This is consistent with the findings of a similar study conducted in South Africa by [22] who reported that women were interested in using injectable because it can be used without their partners' awareness and injectable has less tension than pills like swallowing and remembering of timing of pills swallowing.

Regarding HIV sero-positivity, the majority of HIV positive participants on ART were not influenced by whether to use or not to use family planning due to their HIV Status or the fact that they were on ART medication. Findings from the study showed that women living with HIV/AIDS have fertility desires just like their HIV Negative counterparts and would wish to have at least a child of their own. Although the majority have children, they have hope in getting HIV negative babies despite their status due to programs such as EMTCT. However, these people as well desire to regulate fertility due to the suffering and complications related to raising many children while living with HIV/AIDS. However, in the study conducted by [23] it was noted that being HIV positive status can influence the choice of FP method.

In our study it has been shown that the mean age of PLWHIV using FP were generally young and hence sexually active and they were mainly females who were significantly higher than the males due to better health seeking habits. These results were in concordance with [23]. In another study done in Angola by Prata et al [19], the overall contraceptive prevalence was $55 \%$ for the use of contraceptives, but women aged 25-49 years $(66 \%)$ were more likely to be using modern contraceptives than women aged 15-24 years (48\%). In comparison with this study, the majority of the participants were aged between 27-43 years $(73.8 \%)$ and these used family planning more in comparison to other age groups in this study consistent with the study by Prata and colleagues [19]. 
Kizito et al., Adv. J. Grad. Res.; Vol. 10, Issue 1, pp: 51-61, July 2021

Culture and social beliefs didn't affect FP use with 97\% reported not to have been influenced by these parameters. However, the few who were influenced by culture and social beliefs $(2.7 \%)$ stated that the cultures especially the Baganda believe that FP is an unnatural and is also unaccepted in the Ankole region [24].

The study also showed that the majority of the clients' partners were comfortable with usage of FP $(76.8 \%)$ however the other $23.2 \%$ that were not, stated their reasons being religious beliefs and desire to have more children and feared side effects of the $55.7 \%$ of the participants who used family planning, $50.3 \%$ of the participants' partners were comfortable and $5.4 \%$ of them, their partners were not comfortable even though they were using FP. Of the $44.3 \%$ of the participants who were not using FP, $26.5 \%$ of the participants' partners were comfortable with the use of FP and $17.8 \%$ were not comfortable with the use of FP. Females partners say men with a strict religious background majorly were not comfortable with the partner to use FP and a few of them wished to have more children thus thought family planning hinder the chances of having more children. In comparison with another study done in Jehlak- Sudan by Ahmed et al [20], it reported that the decision regarding utilization of family planning services is one of their concern and men may not have any action regarding that but actually men are the dominant decision maker especially in Jelhak. The study showed that $68 \%$ of the participants stated that the family planning services were easily accessible, it was also noted overall there was improved care and hospitality in the service provision hence improved information about benefits $(82.2 \%)$ and information about risks $(70.8 \%)$.

\section{Conclusion}

Despite the unmet need for FP utilization at TASO Masaka, there has been a more than four-fold increase in the FP service uptake within the last three years from an earlier $14 \%$ uptake to $58.9 \%$ uptake in the current study. This has been attributed to improved accessibility to the service, hospitality by the service providers and the knowledge about the benefits and risks of using FP. All these combined or in isolation have counteracted the negative influence of social and cultural misconceptions about FP. Notwithstanding the improvement of the uptake of FP reported in our study, there is still much effort needed to improve FP uptake and reproductive health seeking habits among PLWHIV.

\section{Declaration}

\subsection{Study Limitations}

Gender bias as most participants were females. The random sampling of the first 10 participants was not followed in the interest of time. We therefore recommend that information about the benefits of using FP among the PLWHIV improves the utilization of the services. Thus, we encourage the service providers to continuously avail information to the community about the benefits of FP use before availing the chosen method. There is a need for continuous monitoring of FP service provision and the effect of FP use on pregnancy incidences among PLWHA enrolled in care and receiving regular FP services. This will help in ongoing assessment of the effectiveness of the strategies being implemented.

\subsection{Acknowledgement}

A special thank you goes to Dr. Ahmed Lwere the Head of Research Habib Medical School (HMS), Faculty of Health Sciences (FHS), Islamic University in Uganda (IUIU). Through our supervisors Professor Fred Kironde and Mr. Hussein Mukasa Kafeero, the content of our study proposal and dissertation report was satisfyingly enriched, so we are highly indebted to them. Special thanks you go to Mr. Ssimbwa Edward, the chairperson research/ CQU committee TASO Masaka and to the special individuals who assisted in the data collection Ms. Nanyondo Victoria, Ms. Matumba Jane and Mr. Kasule Ashraf. Our appreciations also go to the management, staff members and community of TASO Masaka service center where this research was carried out. 


\subsection{Ethical Approval}

The study was a proposal by the Research Ethics Committee (REC) of TASO Uganda; Reference No: TASOREC/089/2020-UG=REC-009.

\subsection{Informed Consent}

The study was conducted according to the guidelines laid down in the Declaration of Helsinki. The participants gave written informed consent to participate and withdraw from the study was voluntary

\subsection{Competing Interests}

The authors of the paper do not have any financial or personal relationship with the other people or organization that could inappropriately influence or bias the content of the paper. The authors therefore declare that they have no competing interests.

\section{How to Cite this Article:}

B. B. Kizito, A. A. . Arigye, S. Namubiru, N. S. . Kabogo, H. M. Kafeero, and F. Kironde, "Family Planning Utilization among PLWHIV Attending TASO Masaka: Evidence from a Cross-Sectional Study", Adv. J. Grad. Res., vol. 10, no. 1, pp. 51-61, Jul. 2021. https://doi.org/10.21467/ajgr.10.1.51-61

\section{References}

[1] WHO, "World Health Organisation," 2020, [Online]. Available: https://www.who.int/news-room/fact-sheets/detail/hiv-aids.

[2] WHO, "World Health Organisation fact sheets about HIV July 2020," [Online]. Available :https://www.who.int/news-room/factsheets/detail/hiv-aids, 2020.

[3] H. Kafeero, D. Ndagire, P. Ocama, A. Walusansa, and S. H, "Sero-prevalence of human immunodeficiency virus-hepatitis B virus (HIV-HBV) co-infection among pregnant women attending antenatal care (ANC) in sub-Saharan Africa (SSA) and the associated risk factors: a systematic review and meta-analysis," Virol. J., vol. 17, no. 1, p. 19, 2020.[Online]. Available: https://link.springer.com/article/10.1186/s12985-020-01443-6

[4] UNAIDS, "UNAIDS data 2020," [Online]. $\quad$ Available https//www.u/naids.org/en/regionscountries/countries/uganda/planning/contraception methods, 2020.

[5] WHO, "WHO June 2019," [Online]. Available: https://www.who.int/news-room/fact-sheets/detail/family-planning-contraception, 2019.

[6] WHO, "2018 Edition of Family Planning: A Global Handbook for Providers," [Online]. Available: https://www.hivsharespace.net/resource/2018-edition-family-planning-global-handbook-provider, 2018.

[7] R. Wilcher, T. Petruney, and W. Cates, "The role of family planning in elimination of new pediatric HIVinfection.," CurrOpin HIV/AIDS., vol. 8, no. 5, pp. 490-497, 2014. [Online]. Available: https://www.ncbi.nlm.nih.gov/pmc/articles/PMC4052828/

[8] L. Chola, S. McGee, A. Tugendhaft, E. Buchmann, and K. Hofman, "Scaling up family planning to reduce maternal and child mortality: The potential costs and benefits of modern contraceptive use in South Africa," PLoS One, vol. 10, no. 6, 2015, [Online]. Available: doi: 10.1371/journal.pone.0130077.

[9] K. Brittain, T. Phillips, A. Zerbe, E. Abrams, and L. Myer, "Long-term effects of unintended pregnancy on antiretroviral therapy outcomes among South African women living with HIV. AIDS.," vol. 33, no. 5, pp. 885-893, 2019. [Online]. Available: https://www.ncbi.nlm.nih.gov/pmc/articles/PMC6528830/

[10] MOH, "Health Sector Development Plan 2015/16 - 2019/20, Ministry Of Health Uganda, 2015," [Online]. Available: https//www.mcsprogram.org/family-planning-key-un-sustainable-development-goals/Family Plan. is Key to UN Sustain. Dev. Goals Posted March 3, 2017, 2015.

[11] L. A. Schrumpf, M. J. Stephens, and N. E. Nsarko, "Side effect concerns and their impact on women's uptake of modern family planning methods in rural Ghana.," BMC Womens. Health, vol. 20, no. 57, 2020. [Online]. Available: https://link.springer.com/article/

[12] S. Babazadeh, P. Anglewicz, J. Wisniewski, P. Kayembe, J. Hernandez, and J. Bertrand, "The influence of health facility-level access measures on modern contraceptive use in Kinshasa, DRC.," PLOS One, vol. 15, no. 7, 2020. [Online]. Available: https: https://journals.plos.org/plosone/article?id=10.1371/journal.pone.0236018

[13] A. Derek, A. Seme, C. Anye, C. Nkfusai, and S. Cumber, "Modern family planning use among people living with HIV/AIDS," Pan Afr Med J., vol. 33, no. 224, 2019. [Online]. Available: https://www.ncbi.nlm.nih.gov/pmc/articles/PMC6814915/

[14] N. Gupta and L. Leite, "Adolescent Fertility Behavior: Trends and Determinants in Northeastern Brazil. International Family Planning Perspectives.," Guttmacher Inst., vol. 25, no. 3, 1999. [Online]. Available: https://www.jstor.org/stable/2991961

[15] A. Dawud, "Contraceptive practices and relative factors: female in predominantly rural Muslims of Northern India.," J. world Heal. Soc. Polit., vol. 5, no. 1, pp. 5-6, 2008. [Online]. Available: https://oda.oslomet.no/oda-xmlui/bitstream/handle/10642/7030/Arhin

[16] L. Kish, "Sampling organizations and groups of unequal sizes.," Am. Sociol. Rev., pp. 564-572, 1965. [Online]. Available: https://www.jstor.org/stable/2091346?casa

[17] B. Nattabi, J. Li, S. C. Thompson, C. G. Orach, and J. Earnest, "Family planning among people living with HIV in post-conflict Northern Uganda: A mixed methods study.," Nattabi, B., Li, J., Thompson, S. C., Orach, C. G., Earnest, J., vol. 5, no. 1, p. $18,2011$. [Online]. Available: https://link.springer.com/article/10.1186/1752-1505-5-18 
Kizito et al., Adv. J. Grad. Res.; Vol. 10, Issue 1, pp: 51-61, July 2021

[18] I. Magala, L. Onega, N. Rose, and S. Patrick, "Factors influencing contraceptive uptake among sexually active HIV positive clients in TASO Masaka, Uganda," J Public Heal. Policy Plan, vol. 1, no. 2, pp. 46-, 2017. [Online]. Available: https://www.rsisinternational.org/journals/ijriss/digital-library/volume-v-issue-i/

[19] K. Ackerson and R. Zielinski, "Factors influencing use of family planning in women living in crisis affected areas of Sub-Saharan Africa: A review of the literature.," Midwifery, vol. 54, pp. 35-60, 2017. [Online]. Available: https://www.sciencedirect.com/science/article/

[20] W. Ahmed, S. Shokai, I. Abduelkhair, and A. Boshra, "Factors Affecting Utilization of Family Planning Services in a Post-Conflict Setting, South Sudan.," AIMS Public Heal., vol. 2, no. 4, pp. 655-6 [Online]. Available: 66, 2015. https://www.ncbi.nlm.nih.gov/pmc/

[21] P. E. Osamor and C. Grady, "Women's autonomy in health care decision-making in developing countries: a synthesis of the literature," Int. J. Womens. Health, vol. 8, no. 191, 2016. [Online]. Available: https://www.ncbi.nlm.nih.gov/pmc/articles/PMC4908934/

[22] L. Hawkins et al., "Integration of Family Planning Services into Healthcare for HIV Positive Women in Botswana.," Int. J. Gynecol. Obstet., 2020. [Online]. Available: https://obgyn.onlinelibrary.wiley.com/doi/full/10.1002/ijgo.13464

[23] S. Magala, I., Onega, L., Rose, N., \& Patrick, "Factors influencing contraceptive uptake among sexually active HIV positive clients in TASO Masaka. Uganda," J Public Heal. Policy Plann, vol. 1, no. 2, pp. 46-9, 2017. [Online]. Available: https://www.rsisinternational.org/journals/ijriss/digital-library/volume-v-issue-i/

[24] A. Nanvubya et al., "Barriers and facilitators of family planning use in fishing communities of Lake Victoria in Uganda.," J. Prim. Care Community Health, vol. 11, 2020. [Online]. Available: https://journals.sagepub.com/doi/full/10.1177/2150132720943775

Publish your books with AIJR publisher-

- $\quad$ Publish with ISBN and DOI.

- Publish Thesis/Dissertation as Monograph.

- Publish Book Monograph.

- Publish Edited Volume/ Book.

- Publish Conference Proceedings

- Retain full copyright of your books.

Submit your manuscript at books.aijr.org
Publish your research article in AIJR journals-

- Online Submission and Tracking

- Peer-Reviewed

- Rapid decision

- Immediate Publication after acceptance

- Articles freely available online

- Retain full copyright of your article. Submit your article at journals.aijr.org 